\title{
"I can guess the month ... but beyond that, I can't tell" an exploratory qualitative study of health care provider perspectives on gestational age estimation in Rajasthan, India
}

\author{
K. Scott ${ }^{1^{*}}$ D, S. Gupta ${ }^{1}$, E. Williams ${ }^{2}$, M. Arthur ${ }^{3}$, U. V. Somayajulu ${ }^{4}$ and L. Noguchi ${ }^{2}$
}

\begin{abstract}
Background: Accurately estimating gestational age is essential to the provision of time-sensitive maternal and neonatal interventions, including lifesaving measures for imminent preterm birth and trimester-specific health messaging.

Methods: We explored healthcare provider perspectives on gestational age estimation in the state of Rajasthan, India, including the methods they use (last menstrual period [LMP] dating, ultrasound, or fundal height measurement); barriers to making accurate estimates; how gestational age estimates are documented and used for clinical decision-making; and what could help improve the accuracy and use of these estimates. We interviewed 20 frontline healthcare providers and 10 key informants. Thematic network analysis guided our coding and synthesis of findings.

Results: Health care providers reported that they determined gestational age using some combination of LMP, fundal height, and ultrasound. Their description of their practices showed a lack of standard protocol, varying levels of confidence in their capacity to make accurate estimates, and differing strategies for managing inconsistencies between estimates derived from different methods. Many frontline healthcare providers valued gestational age estimation more to help women prepare for childbirth than as a tool for clinical decision making. Feedback on accuracy was rare. The providers sampled could not offer ultrasound directly, and instead could only refer women to ultrasound at higher level facilities, and usually only in the second or third trimesters because of late antenatal care-seeking. Low recall among pregnant women limited the accuracy of LMP. Fundal height was heavily relied upon, despite its lack of precision.

(Continued on next page)
\end{abstract}

\footnotetext{
* Correspondence: kscott26@jhu.edu

'USAID's Maternal and Child Survival Program/Johns Hopkins Bloomberg School of Public Health, Baltimore, USA

Full list of author information is available at the end of the article
}

(c) The Author(s). 2020 Open Access This article is licensed under a Creative Commons Attribution 4.0 International License, which permits use, sharing, adaptation, distribution and reproduction in any medium or format, as long as you give appropriate credit to the original author(s) and the source, provide a link to the Creative Commons licence, and indicate if changes were made. The images or other third party material in this article are included in the article's Creative Commons licence, unless indicated otherwise in a credit line to the material. If material is not included in the article's Creative Commons licence and your intended use is not permitted by statutory regulation or exceeds the permitted use, you will need to obtain permission directly from the copyright holder. To view a copy of this licence, visit http://creativecommons.org/licenses/by/4.0/ The Creative Commons Public Domain Dedication waiver (http://creativecommons.org/publicdomain/zero/1.0/) applies to the data made available in this article, unless otherwise stated in a credit line to the data. 
(Continued from previous page)

Conclusion: The accuracy of gestational age estimates is influenced by factors at four levels: 1. health system (protocols to guide frontline workers, interventions that make use of gestational age, work environment, and equipment); 2 . healthcare provider (technical understanding of and capacity to apply the gestational age estimation methods, communication and rapport with clients, and value assessment of gestational age); 3 . client (time of first antenatal care, migration status, language, education, cognitive approach to recalling dates, and experience with biomedical services); and, 4. the inherent limitations and ease of application of the methods themselves.

Keywords: Gestational age estimation, Neonatal health, Preterm birth, India, Qualitative, Client-provider relationships, Quality of care, Antenatal care, Ultrasound

\section{Plain language summary}

Knowing the age of a fetus helps doctors and nurses give appropriate advice and make important healthcare decisions. Yet in many low-resource settings, doctors and nurses struggle to accurately estimate fetal age. This study asked 30 nurses, doctors, and policy experts in India about how fetal age is estimated and used in the government sector. We found that healthcare providers relied primarily on calculating fetal age based on the pregnant woman's last menstrual period (LMP) or by measuring fundal height (size of the pregnant belly), but they struggled with low client recall of LMP and reported using their hands rather than measuring tapes for fundal height measurement. Early ultrasound, which is the gold standard for estimating fetal age, was rarely used because pregnant women sought healthcare too late, found ultrasounds difficult to access, and considered them a low priority. Strategies used for estimating fetal age varied widely from provider to provider. Many doctors and nurses were overconfident about their capacity to make accurate estimates, and only a minority recognized the inherent limitations to the estimation methods. We present a framework of factors at the health system, healthcare provider, client, and method/ tool levels that must be improved to make fetal age estimation and use more successful. Strategies include increasing the health system's capacity to actually use these estimates to help women and infants, simplifying documentation, improving healthcare provider training and supervision (including on client-provider communication), encouraging newly pregnant women to visit healthcare workers as early as possible, increasing ultrasound accessibility, and improving women and health workers awareness of the value of accurate estimates.

\section{Background}

13.7 million preterm births (births prior to 37 weeks' gestation) occur annually in low- and middle-income countries (LMICs) - over 90\% of the global burden [1]. Preterm birth complications are the leading cause of mortality among children under five years [2]. Of the 2.7 million annual neonatal deaths (occurring in the first 27 days), 0.94 million (35\%) are attributable to conditions related to preterm birth [2] [3]. In India, approximately 3.5 million infants are born too early each year [1] and preterm birth-related conditions are the cause of 0.3 million deaths among Indian children under five [2]. Conditions related to preterm birth cause $43 \%$ of all neonatal mortality and $27 \%$ of all under five mortality in India [2].

Knowing the gestational age of a fetus is vital to the provision of antenatal corticosteroids to women at risk of preterm delivery, which could save 370,000 lives each year [3]. While the administration of antenatal corticosteroids is recommended for women at risk of preterm birth from 24 weeks to 34 weeks, administration after 34 weeks' gestation brings risk of harm to mature fetuses exposed to corticosteroid in-utero that may outweigh the benefits [4]. The World Health Organization thus recommends that: "antenatal corticosteroid should not be routinely administered in situations where the gestational age cannot be confirmed" [4].

Accurate gestational age assessment also enables the timely initiation of appropriate care for a preterm infant. Furthermore, many aspects of maternal healthcare rely on gestational age estimates, including mobile phonebased health messaging programs that provide stagebased pregnancy information to millions of women in LMICs $[5,6]$.

In high-resource settings, early pregnancy ultrasoundbased dating is standard practice and considered the most accurate method of gestational age estimation. The gold standard is ultrasound prior to 14 weeks gestation, while estimates prior to 22 weeks are considered adequate [7]. Ultrasound-based dating during the first trimester often uses crown-rump length to estimate gestational age, since there is a linear relationship between this measurement and gestation age during early pregnancy [8]. Later in pregnancy, gestational age can be estimated, albeit with lower accuracy, through ultrasound measurement of combinations of biparietal diameter, head circumference, abdominal circumference and femur (diaphysis) length [9]. Menstrual-based dating is also widely used, and calculates the estimated date of full 
term delivery ( 40 weeks' gestation) as 280 days from the first day of a woman's last menstrual period (LMP) [10], but is only accurate among women who menstruated regularly and can recall their LMP.

In low-resource settings, gestational age estimates are often inaccurate [11], making it difficult for health care providers to take appropriate clinical decisions. Little is known about how health care providers in these settings determine estimated date of delivery, their use of and confidence in various gestational age estimation methods, and their use of gestational age estimates for identifying and managing induction of labour, imminent pre-term birth, post-term and complicated pregnancies, and preterm neonates. Systems for documenting and using gestational age estimates throughout pregnancy are also under-researched, despite their importance in enabling gestational age estimates to inform the provision of time sensitive interventions.

Measuring fundal height is one low-cost approach used in low-resource settings [12]; however, it only generates approximate gestational age estimates, is dependent on provider skill, and does not differentiate between growth restriction and lower gestational age - a particularly important issue in regions with endemic malaria, as malaria is strongly associated with intrauterine growth restriction [13]. Even when multiple (up to seven) fundal height measurements are entered into population-specific models accounting for fundal height and slope (gradient), researchers have found this gestational age estimation method produces too great a range to enable the classification of prematurity [14].

This paper presents qualitative findings on the practice of gestational age estimation, documentation, and utilization for clinical care of pregnant women in lowresource health facilities in Rajasthan, India. The aim of this research is to document current systems and protocols in order to identify facilitators and barriers to accurate gestational age estimation, appropriate documentation of gestational age estimates, and correct use of gestational age estimates to provide appropriate gestational agespecific interventions during pregnancy and delivery.

\section{Methods}

\section{Study setting}

Research took place in Rajasthan, a state in northwestern India with a population of 78 million. Rajasthan was selected in consultation with the Ministry of Health and Family Welfare, as part of its focus on improving maternal and newborn health care in Empowered Action Group states. While $91 \%$ of households had some (often limited and irregular) access to electricity, only $45 \%$ had improved sanitation [15]. Male literacy was $85 \%$, female literacy was $57 \%$ and only $25 \%$ of women had 10 or more years of schooling [15]. In 2015, $63 \%$ of pregnant women received antenatal care (ANC) in the first trimester and 38\% received the recommended four ANC visits [15]. The institutional birth rate was 84 , and $64 \%$ of institutional births were in a public facility [15]. The total fertility rate was 2.4 children per woman and infant mortality was 41 per 1000 live births [15]. Rajasthan continues to experience endemic malaria [16].

\section{Data collection}

The study consisted of 30 in-depth interviews with frontline ANC providers $(n=10)$, intrapartum care (IP) providers $(n=10)$ and expert key informants $(\mathrm{KI})$ in the government (public) health sector $(n=10)$ (Table 1$)$. Our sampling was guided by the principle of thematic saturation for the healthcare providers [17], while key informants were sampled based on access and availability across a pre-set range of profiles. We note however that the ANC and IP classifications are not rigid and merely indicate the focus of each respondent's work because many ANC providers also conduct deliveries and all IP providers at least occasionally provide ANC.

Respondents were located in rural health sub-centers $(n=8)$, rural primary health centers $(n=8)$, district hospitals $(n=8)$, nursing colleges $(n=3)$ and ministry or department of health offices $(n=3)$. ANC providers, which included auxiliary nurse midwives (ANMs) $(n=7)$, a staff nurse $(n=1)$ and a medical officer $(n=1)$, are the first point of contact in the government health system for pregnant women. They determine the initial gestational age estimate and generate a woman's pregnancy-related documents. Intrapartum care providers, which included ANMs $(n=2)$, staff nurses $(n=3)$, a lady health visitor $(n=1)$ and medical officers $(n=4)$, make use of gestational age estimates and ANC documents to provide care throughout labour and delivery; many of them also conduct some antenatal care. They also assess and refer complex cases to higher level facilities. Key informants, which included medical officers in supervisory and tertiary care roles $(n=4)$, government health program coordinators and officers $(n=3)$, and teachers in nursing colleges $(n=3)$, provided macro level perspectives on gestational age estimation as educators, experienced and senior healthcare providers, or as policymakers working in maternal and child health.

Data were collected by three qualitative researchers (two female and one male) after a two-day training in Delhi; all three had experience in public health qualitative research but none were trained in nursing or paramedic subjects. Training included orientation to the clinical components of gestational age estimation. Data collection took place between January and April 2018 in five districts of Rajasthan state (Udaipur $(n=6)$, Bundi $(n=3)$, Pratapgarh $(n=5)$, Bharatpur $(n=10)$, Swaimadhopur $(n=2))$ and the national capital, Delhi $(n=4)$. 
Table 1 Qualitative respondents and their profiles

\begin{tabular}{|c|c|c|c|c|c|}
\hline Respondent group & Respondent title & Profile & Male & Female & Row total \\
\hline \multirow[t]{2}{*}{ Antenatal care provider } & Auxiliary nurse midwife (ANM) & $\begin{array}{l}\text { Female maternal and child health worker with } 1.5 \text { years } \\
\text { training ( } 6 \text { months midwifery focused) }\end{array}$ & 0 & 9 & 9 \\
\hline & Lady health visitor & $\begin{array}{l}\text { ANM with } 5+\text { years' experience and } 6 \text { months additional } \\
\text { upgrade training }\end{array}$ & 0 & 1 & 1 \\
\hline \multirow[t]{2}{*}{ Intrapartum care provider } & Staff nurse & $\begin{array}{l}\text { Registered nurse with } 3 \text { (General Nurse Midwife) or } 4 \\
\text { (BSc Nursing) years training ( } 6 \text { months midwifery focused) }\end{array}$ & 1 & 3 & 4 \\
\hline & Medical officer (MO) & $\begin{array}{l}\text { Bachelor of Medicine, Bachelor of Surgery (MBBS) doctor, } \\
5.5 \text { years training }\end{array}$ & 5 & 1 & 6 \\
\hline \multirow[t]{6}{*}{ Key informant } & $\begin{array}{l}\text { Medical officer: senior, } \\
\text { principle, or in-charge }\end{array}$ & MBBS doctor in a supervisory role at district level hospital & 1 & 3 & 4 \\
\hline & Technical coordinator & $\begin{array}{l}\text { Ministry of Health and Family Welfare (MoHFW) program } \\
\text { and technical expert staff }\end{array}$ & 1 & 0 & 1 \\
\hline & $\begin{array}{l}\text { Nursing college principal } \\
\text { or vice principal }\end{array}$ & Nurse now working in the education sector & 0 & 3 & 3 \\
\hline & $\begin{array}{l}\text { Reproductive and child } \\
\text { health officer }\end{array}$ & District level government health program officer & 1 & 0 & 1 \\
\hline & $\begin{array}{l}\text { Maternal and child health } \\
\text { head }\end{array}$ & District level government health program officer & 1 & 0 & 1 \\
\hline & & Column total & 10 & 20 & 30 \\
\hline
\end{tabular}

Interviews ranged from 38 to $67 \mathrm{~min}$ and were conducted in Hindi then translated and transcribed into English.

The interviews explored a range of domains, including: the respondent's work environment (client load, relationships, supervision); typical gestational age estimation methods used and their perceived accuracy; issues around late ANC care-seeking; challenges to determining accurate gestational age and strategies to achieve accurate gestational age estimates; how gestational age estimates are used during antenatal and intrapartum care, including the identification and management of pre-term labour and infants; gestational age-related training received; gestational age-documentation practices; and recommendations for improving gestational age estimation, documentation, and use (the ANC interview guide is provided in Annexure 1).

\section{Data analysis}

Analysis began with an initial review of five transcripts and the development of a codebook. The codebook consisted of 176 sub-codes developed from a priori areas of interest as well as topics emerging from the data. These sub-codes were grouped into 34 main codes based on the a priori interests of the study. For example, we had an a priori interest in reasons for late ANC care-seeking among pregnant women, so when respondents spoke about migrant workers sometimes not coming to the health centre until the very end of their pregnancy we created the sub-code "migrant labourers, those who leave the village" and grouped it under the main code "late ANC/low care-seeking." Transcripts were coded in the qualitative data management software Dedoose (Los Angeles, CA). Code reports were generated by listing all text from the transcripts that were "tagged" with a specific sub-code, along with characteristics of the respondents who made each statement. The code outputs were read for each topic and salient overarching themes were identified guided by thematic network analysis [18]. Each theme is described in the findings section below, and example quotations from the transcripts are provided. Finally, we synthesized the thematic findings into a framework that summarizes the factors influencing gestational age estimation, documentation and use (Fig. 1, presented in the discussion).

\section{Ethics}

Ethical approval was received from the Johns Hopkins Bloomberg School of Public Health Institutional Review Board and Sigma, India. All respondents provided written informed consent before participation.

\section{Results}

We first provide an overall picture of the gestational age estimation situation in rural Rajasthan, including details on the use of the specific estimation methods (LMP, fundal height and ultrasound). We then report findings on the documentation and use of gestational age estimates in the government health sector. We finish with provider comments on potential avenues to improving gestational age estimate accuracy. Respondents are identified by a code indicating whether they primarily provided intrapartum (IP) or ANC as well as their serial number. 


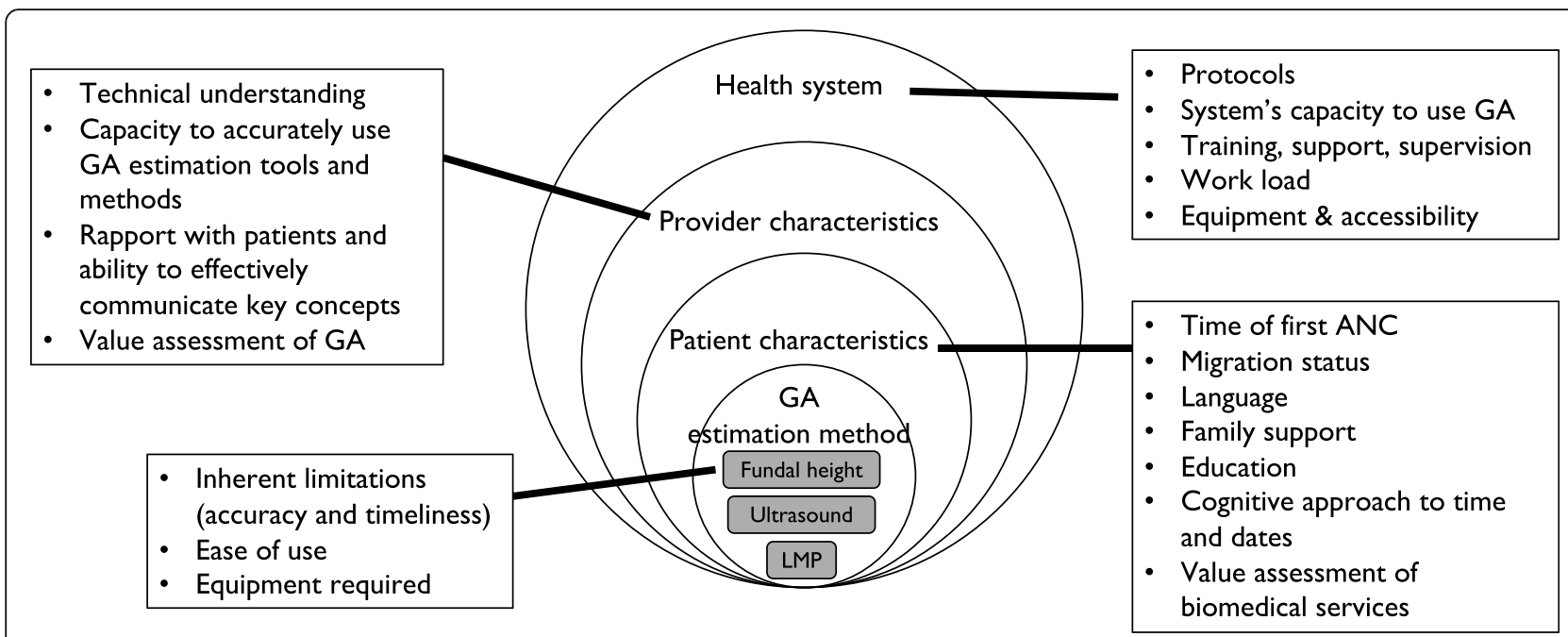

Fig. 1 Factors influencing gestational age estimation, documentation, and use

Lack of clear and consistent protocol for gestational age estimation

Providers discussed using a combination of LMP, fundal height, and ultrasound to determine gestational age. However, they also had widely varying assessments of the relative use and accuracy of the different methods. Thirteen of the 20 respondents involved in day to day ANC or intrapartum care in the government (public) sector described estimating gestational age through assessing LMP and fundal height but placed different weights on the relative importance and accuracy of the two. Six respondents (IP01, M, MO; ANC10, M, staff nurse; ANC08, F, ANM; IP02, F, staff nurse; IP03, F, lady health visitor; IP04, F, staff nurse) generally relied on LMP and fundal height equally.

[We calculate gestational age] by checking the abdomen through fundal height, and according to the time mentioned by the women we use LMP. (ANC10, M, staff nurse)

Four providers (ANC03, F, ANM; ANC05, F, ANM; ANC07, F, ANM; IP06, F, MO) relied on LMP but checked fundal height as a secondary confirmation.

We ask her the LMP and accordingly we note down her EDD [estimated date of delivery]. And we tell her the date. After that once again we do the fundal height test to be sure or in case that date has elapsed. (ANC07, F, ANM)

One provider (IP10, M, MO) said that LMP, fundal height and ultrasound were used equally to determine gestational age, although ultrasound was most accurate, and two providers (IP08, F, ANM; ANC04, F, ANM) described using fundal height only when a woman could not recall LMP:

First we ask the LMP date during the ANC, if they tell us then we calculate the EDD. However, if they are not able to tell their LMP then we calculate by fundal height. (IP08, F, ANM)

Among the seven respondents who did not describe primarily using both LMP and fundal height, five spoke only of using LMP, or heavily emphasized LMP (ANC01, M, MO; ANC02, F, ANM; ANC06, F, ANM; IP07, F, ANM; IP09, F, staff nurse) and two said they relied solely on fundal height (ANC09, M, MO; IP05, $\mathrm{M}, \mathrm{MO})$. We asked providers what they would do if LMP and fundal height indicated different estimated gestational ages. Most said they would ask the woman to have an ultrasound, while also explaining that few women actually go to have ultrasound examinations done (discussed in the sub-section on ultrasound, below).

Many healthcare providers expressed high confidence in the accuracy of their methods; only a minority reflected on the limitations inherent to these methods:

[Back when I conducted deliveries] the EDD was almost correct; there was only a difference of 1-2 days. (IP07, F, ANM)

In case the woman has come for her delivery, we will get to know how many months pregnant she is. With the kind of experience we have, we are able to make an assumption as to when the women had her last period and how many months pregnant the woman is. (IP04, F, staff nurse) 
We give them almost perfect date after abdominal exam. (IP03, F, lady health visitor)

[ ... ] we calculate their LMP and it comes accurate and only difference comes of five days or ten days. (ANC02, F, ANM)

There is no 100 percent accuracy. There can only be an estimate; the date of delivery can vary from 7 to 10 days before or 5 days after the expected date. We can just make an assumption that the delivery is expected to happen near a calculated date. (IP10, $\mathrm{M}, \mathrm{MO}$ )

We get the exact [EDD] from the fundal height. And we can't tell it accurately through LMP. Mostly have come around one month difference or 4 month, 4 week difference comes. (ANC09, M, MO)

It is noteworthy that many providers spoke about gestational age in terms of months, rather than weeks - potentially indicating acceptance or recognition of low precision.

\section{Last menstrual period: preferred method but difficult to assess}

When probed on their preferred method for assessing gestational age, almost all providers explained that they would ideally use LMP but resorted to using fundal height and sometimes ultrasound because women could not recall their LMP. Providers explained that most of their female clients in rural Rajasthan understood time in relation to harvests and festivals, rather than months and days.

We ask LMP. Ladies were not so much aware about LMP [ ... ] They said that it is happens at the harvest time, when there was no moon, full moon was there. Accordingly, we have to calculate LMP. We have to face many problems like in which month and date the moon was? When was the harvest? We have to calculate accordingly because they are uneducated people and will not understand. Accordingly, we calculate LMP. That is why differences occurs in LMP. (ANC03, F, ANM)

While some providers felt quite confident that they could derive an accurate LMP from harvest events, festivals or moon cycles (e.g., ANC03, F, ANM), others lamented moving from rough time periods to a specific estimate, noting a limitation in accuracy (e.g., ANC07, F, ANM). Overall, we found a very wide range in provider estimates of the percentage of women who could accurately recall LMP. Despite working with similar client populations, some providers said that only $10 \%$ of women recall LMP, while others said $20,5080 \%$ or almost all (98\%) women recall it. Providers found moving from LMP to EDD straightforward; they easily recalled the formula of 9 months plus 7 days, and noted that all ANC registers had a calendar or chart to help them calculate and that during data entry the EDD was generated by the computer from the LMP.

Interviewees identified the following client profiles as most unable to report a precise LMP: Uneducated women, tribal women (adivasis, who were considered very shy about menstruation), Muslims (Mewat/Meo), people with many children and frequent pregnancies (because, according to some providers, they are careless and uneducated), older women (who generally have low education and are also shy about the fact that they remain sexually active), newlywed women (also very shy about anything to do with sex or menstruation), and women who speak a regional dialect rather than Hindi (because they struggle to communicate with their Hindispeaking healthcare providers).

While some providers spoke of working with their clients to move from recalling menstruation in relation to festivals or the lunar cycle to identifying a date on the Gregorian calendar, most providers could not describe any way of putting women at ease or asking follow up questions to try to access a more accurate estimate of LMP. Two providers (ANC09, M, MO, ANC04, F, ANM) and one teacher in a nursing college (KI02, F, principal of nursing college) mentioned asking the woman's mother-in-law, sister-in-law, or community health worker (ASHA or anganwadi) to help recall her LMP. When asked about strategies to get accurate LMP and reasons why LMP may be inaccurate, no providers noted that they confirmed that the woman understood that they were seeking the first day of her LMP rather than middle or last day, none confirmed that women were recalling LMP and not their first missed period and only one provider (KI07, F, MO, district hospital) mentioned checking that the respondent had a regular period.

A senior medical officer (KI07) explained several ways through which she worked with her clients to access a best-possible LMP:

We try to talk to her in her language. Like we ask her, "When was the time you used the cloth last time?" Then she tells the time in accordance to the full moon, half-moon or no moon; we ask her the details in the way she can answer. Then we calculate the date. We ask her if her menses were regular. We ask her the duration of her menses. (KI07) 
Very few providers noted reasons why LMP may not be an appropriate gestational age assessment mechanism for all women. Being unable to report LMP was attributed to women's inability to recall this information, with few providers noting that some women may have irregular periods (KI07, senior medical officer), mistake spotting for menstruation (KI01, technical coordinator, MoHFW), be on a birth control that stops menstruation (ANC08, F, ANM, ANC07, F, ANM), get pregnant after a prior pregnancy and before resuming menstruation, or think that missed periods are due to menopause rather than pregnancy (ANC07, F, ANM).

\section{Fundal height: an assessment in the healthcare provider's hands}

In a context of low confidence in LMP and poor ultrasound accessibility, fundal height offered one form of gestational age estimation that was always available to healthcare providers. Fundal height was frequently - but not universally - assessed during each antenatal care checkup after the first trimester. While some providers gave detailed descriptions of their method of calculating gestational age from fundal height and were confident in their capacity (ANC03, F, ANM; ANC09, M, MO), others were vague or unsure (KI10, M, MCH head, district hospital). The use of hand- or finger-based estimates rather than measuring tapes was commonly reported. No providers discussed client characteristics that could influence fundal height, such as the woman's weight.

R: If the fetus or fundus is near the navel then the woman is 7 months pregnant. If it's below the navel then the fetus is 5 months old. [I measure] by the palm of my hands. So how much above or below the fetus is; we get to know the gestational age of the fetus. If it is hollow underneath the navel and the fetus comes down at the lower abdomen, then it is the ninth month. But the fundal height method does not confirm the exact date of delivery like it does in the LMP method. I can't tell the fixed days by that [fundal height] method. I can guess the months but beyond that, whether it is the starting of 5 or 6 th month or end; I can't tell that.

\section{I: Have you ever performed it on anyone?}

R: I have done it but not much. Only when they are not able to tell their LMP. (IP08, F, ANM)

Providers also assessed fundal height to track fetal growth, noting that a small fundus could indicate growth restriction while a large fundus could indicate twins or other issues. The fact that these dual uses of fundal height assessment were at odds was only discussed by one respondent (ANC10, $\mathrm{M}$, staff nurse).

Providers reported that women were generally comfortable with the physical examination of their abdomen, noting that they "are quite keen on getting it done" (ANC06, F, ANM) because it reassures them that the fetus is developing well and that women "feel satisfied that they got checked" (ANC08, F, ANM). Two respondents (ANC09, $\mathrm{M}, \mathrm{MO}$ and $\mathrm{ANC10}, \mathrm{M}$, staff nurse) noted gender considerations, specifying that women prefer a female healthcare provider or that male doctors are sure to keep a female attendant in the room while checking fundal height.

\section{Ultrasound: inaccessible and few noted importance of early examination}

Provider recommendations on when ultrasound is required, how often, and whether it is needed vary widely. Most ANC providers primarily recommended ultrasound during pregnancy in cases of bleeding, pain, a history of miscarriage, or suspected complications. Some providers said it should be routinely performed in the second or third trimester to check for abnormalities. Most, but not all, providers reported that they sent women for ultrasound examinations if they could not recall LMP or when LMP and fundal height suggested different gestational age estimates.

Sometimes few of them forget their LMP; through ultrasound we can find gestational age. (ANC05, F, ANM)

Because some ladies are not able to tell their LMP date we advise them to get ultrasound done so that they will get to know exactly about the baby. (ANC02, F, ANM)

Women get their ultrasound done on their own. [ ... E Everyone does not get their ultrasound done. We only suggest getting an ultrasound done when we see there are complications. I don't suggest everyone for it. Many of them are not in a position to get it done. (ANC07, F, ANM)

While three providers (IP06, F, MO; IP03, F, lady health visitor, IP01, M, MO) noted that ultrasound during the first trimester enabled accurate gestational age estimation, two respondents (KI01, technical coordinator, MoHFW and ANC06, F, ANM) said that ultrasound should not be done during the first trimester. Providers noted that women were increasingly coming for their first ANC checkup in the first trimester, but that late ANC remained an issue for women living in conservative households (particularly those practicing purdah, the physical segregation and veiling of women), migrants, and older women who already have several children. 
While asking LMP and measuring fundal height could be done by the providers themselves, most could only suggest that women go for ultrasound examinations, since ultrasound facilities were only available in higher level facilities or private clinics. Most providers said that few women had any ultrasound done during pregnancy; however estimates of the percentage of women having ultrasound ranged from 5\% (IP05, M, MO) to 30\% (IP10, M, MO) to $90 \%$ (IP04, F, staff nurse). Having an ultrasound requires spending money in the private sector or waiting for many hours in the public sector. Additional reasons for low uptake included that women were discouraged by older family members, could not find anyone to accompany them (and were not able to travel alone due to social norms); did not want to or could not leave the house; could not afford the cost including for transportation; and a prevailing sense that ultrasound would be a waste of time and money in normal uncomplicated pregnancies.

Nobody wants to get the ultrasound done over here in the village. Even if they want to they can't because they don't have enough money. The elderly people say,

"No point getting it done." (IP07, F, ANM)

Confidence in the capacity of ultrasound to enable accurate gestational age estimation varied, with some (ANC06, F, ANM, ANC09, M, MO, IP05, M, MO and ANC03, F, ANM) saying it is highly accurate and/or the preferred gestational age estimation method and others (ANC07, F, ANM; IP09, F, staff nurse; IP04, F, staff nurse) considering ultrasound to be inaccurate. A nursing college teacher was unsure: "I'm quite unsure whether the ultrasound is that much reliable or not" (KI02, F, principal of nursing college).

Ultrasound is very useful to us. Estimation of EDD by ultrasound is accurate. [ ... ] [We use ultrasound] after the completion of 3 months. And in sixth month if a woman faces any problem [ ... ] (IP05, $\mathrm{M}, \mathrm{MO})$

But mostly the EDD that is proposed by the ultrasound is inaccurate. It is only one or two percent where the dates are correct. At times the delivery happens 15 days before the date proposed by ultrasound; at times it is 10 days post the date proposed.

(ANC07, F, ANM)

\section{Challenges in the work environment and non-specific understandings of the clinical benefit of accurate gestational age}

Providers noted their high workload and overcrowding in health facilities as a barrier to accurate record keeping and proper gestational age estimation: "Due to workload pressure sometime it feels difficult to complete it the ANC register]" (ANC04, F, ANM). They reported initial or refresher medical education on gestational age estimation, but none had received training on how to handle instances when gestational age estimates from different sources did not match or on when gestational age estimates should be revised. Multiple records were created and maintained (e.g., ANC book which is kept with the ANM, an ANC registration form which is sent to the higher level health facility, a Mamta card which the pregnant woman keeps with her, an Anganwadi record), which created a high documentation burden.

Some providers were frustrated by their "uneducated" women (usually in reference to Muslim and tribal women) who did not abide by medical advice, particularly for family planning and immunization. Some providers struggled to communicate with their female clients because the women spoke a regional dialect. Both male and female healthcare providers reported that some women were too "shy" and were difficult to communicate with about LMP or sexual activity.

Respondents noted that women often forgot their ANC cards and ultrasound reports for future ANC visits and at time of delivery because they were in a hurry, and labour pain made it difficult to think of anything else, they were not aware that the records were important and women's in-laws and husband do not "take care" about these documents (IP06, F, MO). Intrapartum care providers reported that although they might check LMP in the client's ANC card, if the woman brought it with her, ANC documentation was primarily used for record keeping rather than for clinical decision making at the time of delivery. Instead, providers conducted their own examinations at time of delivery and asked the woman again for her LMP and EDD.

At last moment documentation is not necessary. During delivery the patient is tackled first, her condition is examined, we check whether labour is absent or we have to induce labour. (KI01, technical coordinator, MoHFW)

Even if the ANM gives them the monthly record card, still the patient will have to remember their dates for the period and note them down or tell the ANM or us. (IP10, M, MO)

Interviewer: What is the purpose of those documents?

Respondent: They are used for maintaining records. (ANC10, M, staff nurse)

Intrapartum care providers report that women's ANC records tended to be incomplete. They attributed gaps 
in records to: pregnant women coming irregularly for ANC; women not knowing or having key data, such as lacking a marriage certificate, Bhamashah (health insurance) number, or bank account; communication barriers (shyness, linguistic issues); high health provider workload; and frontline providers making general estimates of LMP, blood pressure and other parameters.

We asked antenatal and intrapartum care providers about the value of gestational age estimation. Only four of 20 spontaneously noted that gestational age could be a criterion for clinical decision making around the time of labour. Most providers focused on gestational age as generally useful for enabling women and their families to prepare for the baby's birth, and for tracking fetal growth. One mentioned that gestational age enabled them to tailor advice based on the trimester (e.g., when to take iron and calcium supplements, to rest and come for frequent checkups in the final month) (ANC09, M, $\mathrm{MO}$ ), three mentioned that gestational age was used to intervene in cases of post-term pregnancy (ANC05, F, ANM, IP06, F, MO; IP09, F, staff nurse), one mentioned that it enabled them to know that a baby was preterm (IP09, F, staff nurse).

On probing, all intrapartum care providers reported the use of corticosteroid drugs (dexamethasone) in cases of immanent pre-term birth. When asked how they identified instances of pre-term labour, they mentioned using some or all of the following, depending on what information was available: the pregnant woman's telling them that she is only seven or 8 months pregnant but experiencing labour pain, the LMP and EDD written on the client's chart, the size of her abdomen, and any ultrasound reports provided.

They will see the Mamta Card, LMP, EDD and the ultrasound report. They will ask the patient how many months pregnant she is. After checking all this they will know [if it is a case of pre-term labour]. (IP04, F, staff nurse)

When the patient is in labour and the EDD and fundal height are noted to be less, then we get to know about it [preterm labour]. Every woman gets to know when it has only been 8 months or 7 months. (IP01, M, MO)

Interviewer: How would you discover that it is a preterm birth?

R: Either by the report or by looking at her stomach as the stomach will be very small as compared to those of full term pregnancies. Also by checking the LMP, EDD and ultrasound reports, we can tell.
I: What documents do you check for their EDD?

R: Mamta card and ultrasound report. And the woman also tells that she is so and so months pregnant or that her EDD was that of a future date but she is having the pain now.

I: Is there any other way to find out?

$R$ : The woman will be considerably weaker, she will be anemic. (IP04, F, staff nurse)

\section{Ideas for improving gestational age estimation and documentation}

When asked how gestational age estimation could be improved, providers primarily focused on educating women about the importance of remembering LMP and suggested that community health workers (called ASHA sahyoginis or anganwadi workers) could conduct additional outreach to encourage early ANC.

We should ask a woman to have regular antenatal check-ups, through which she will also stay updated about her date. According to me if a woman is regular in her ANC check-ups she will not face any complication during delivery period. (IP06, F, MO)

Six healthcare providers called for improved availability and uptake of ultrasound scans. Four wanted additional training for frontline workers on gestational age estimation, and one (ANC09, M, MO) specifically asked to be trained on how to properly assess fundal height. While some providers said that the forms used to document ANC were easy and required no changes, one medical officer said that the ANC cards (Mamta cards) are poorly designed. Three providers expressed excitement about moving to digital record keeping, suggesting that this would reduce data entry errors. Several medical officers suggested that the oversight and training of ANMs needs be strengthened, including ensuring that ANMs complete ANC records accurately (IP05, M, MO) and that ultrasound technicians are sufficiently skilled.

\section{Discussion}

Health care providers in rural Rajasthan reported determining gestational age and estimating date of delivery using some combination of last menstrual period, fundal height, and ultrasound. Their description of their practices showed a lack of standard protocol, varying levels of confidence in their capacity to make accurate estimates, and differing strategies for managing inconsistencies between estimates derived from different methods. We found that many frontline healthcare providers valued gestational age estimation because it could help 
women prepare for childbirth, but not as a tool for clinical decision making. Providers also tended to report overconfidence in the accuracy of their gestational age estimations, with only a few discussing the limitations inherent to the methods or the potential inaccuracies introduced by features of their work environment.

Providers faced a number of barriers to making accurate estimates. Accurate ultrasound-based dating was unlikely because many women received their first ANC in the second trimester and because ultrasounds required women to travel long distances, pay (in the private sector) or wait for many hours (in the public sector) and were considered unnecessary by most women and providers in normal healthy pregnancies. Health care providers considered last menstrual period to be an affordable and accessible method of estimating gestational age during pregnancy, but were limited by low client recall. In addition, providers themselves tended to assume that all women had regular menstruation prior to becoming pregnant. While fundal height assessment was popular - and was the only method that healthcare providers could themselves control completely -providers used their hands rather than measuring tapes to generate estimates rounded to months rather than weeks gestation.

Our research enabled the development a framework to understand factors that influence the accuracy, use and documentation of gestational age estimates, which identifies influences across four levels: health system, provider characteristics, client characteristics, and estimation method (Fig. 1). This framework can help policymakers, academics, and healthcare workers identify areas for intervention, as discussed below. Furthermore, this framework could help stakeholders consider the holistic environment in which gestational age estimation occurs, which will in turn enable them to identify weakness and understand how the spheres interact to build a culture around gestational age estimation, documentation and use.

Health system policymakers can consider interventions to improve gestational age estimation at multiple entry points of this framework (Table 2). Many of these interventions could be integrated within India's National Health Mission, including its guidelines and norms for government health care facilities and staff $[20,21]$ and the Janani Surakha Yojana conditional cash transfer program, which is associated with more women receiving ANC and more providers conducting abdominal examinations during ANC [22].

Our study points to the need for improved access to ultrasound for gestational age estimation coupled with improving demand generation for early ANC, since so few women confidently recalled their LMP and since fundal height enables only approximate dating. However, there are tradeoffs and potential drawbacks to the increased use of ultrasound in low resource health systems [23]. Access to ultrasound only contributes to more accurate gestational age estimates if the machines produce sufficiently high quality images, which is not the case for many portable machines [23], and if providers are appropriately skilled and only use ultrasound for gestational age estimation within the recommended time period, which has been found to often not be adhered to [24]. More broadly, a randomized controlled trial in Pakistan, Kenya, Zambia, Democratic Republic of Congo and Guatemala found that increased access to ultrasound was not linked to improved client outcomes.

Given the challenges with widespread early access to ultrasound, gestational age estimation using last menstrual period remains a viable option for frontline health workers in low resource settings. However, this method yields accurate estimates only among women who had regular menstruation prior to pregnancy, something frontline healthcare providers may not account for. The proportion of pregnant women in low resource setting who have irregular or absent periods prior to pregnancy, which proscribes the use of LMP for gestational age estimation, is likely high due to undernutrition, hormonal disorders, early menopause, becoming pregnant again before postpartum menses regulates, and the use of many forms of contraception. Even among women who were menstruating regularly before becoming pregnant, communication about LMP is hindered by low client recall, poor client-provider rapport [25], shame around menstruation [26, 27], and traditional approaches to timekeeping (harvests, festivals). Efforts to reduce shame and improve women's reproductive health knowledge may indirectly improve the use of LMP for gestational age estimation.

Late ANC reduces the likelihood that a woman will accurately recall the date of her last menstrual period and limits the capacity for ultrasound to produce an accurate gestational age estimate. ANC after the first trimester is common in many low and middle income countries, particularly among rural areas and among less educated women [28-30]. Cultural norms around shame or bad omens influence when the news of a pregnancy can be shared with one's community: first ANC is delayed among women who wait until pregnancy is visible before talking to others, including health workers, often linked to an understanding that early pregnancy is a period of vulnerability [31, 32]. Women in South Africa [33] and India [34] have explained that they consider pregnancy a natural process with little risk to one's health (as opposed to labour, which has clear health risks), and thus does not require multiple or early ANC. Interventions have been moderately successful at changing these norms to increase ANC uptake through participatory 
Table 2 Potential entry points for improving gestational age estimation

\begin{tabular}{|c|c|}
\hline Level & Potential intervention points to improve gestational age estimation, documentation, and use \\
\hline Health system & $\begin{array}{l}\text { - Clarify protocols and expectations on which gestational age estimation methods to use and when, how to } \\
\text { navigate conflicting estimates, and how to document and use gestational age } \\
\text { - Increase health system focus on the risks of preterm birth, and the importance of identifying preterm births } \\
\text { quickly in order to provide supportive interventions } \\
\text { - Build health system capacity to use gestational age estimates for clinical decision making, to ensure that } \\
\text { gestational age accuracy is a meaningful part of antenatal and intrapartum care provision (i.e., ensure that } \\
\text { interventions are actually available to address problems identified through gestational age estimation) } \\
\text { - Ongoing quality improvement: Train, support, and supervise health workers on gestational age estimation, } \\
\text { including training that compares provider estimates to a gold standard and offers feedback } \\
\text { - Reduce provider workload so that they have sufficient time to assess and document gestational age during ANC } \\
\text { - Increase the accessibility of equipment that aids gestational age estimation, particularly ultrasound but also } \\
\text { measuring tapes for fundal height assessment and gestational age wheels }\end{array}$ \\
\hline $\begin{array}{l}\text { Frontline health care provider } \\
\text { characteristics }\end{array}$ & $\begin{array}{l}\text { - Improve providers' technical understanding of gestational age estimation methods, including the inherent } \\
\text { limitations of each method and the standardized protocols (such as ACOG [19]) for resolving discrepancies between } \\
\text { LMP and ultrasound dating } \\
\text { - Improve providers' capacity to correctly estimate gestational age, including on how to work with women to get a } \\
\text { best-possible last menstrual period date when possible, clarifying that estimation is from the first day of the last } \\
\text { menstrual period, training on how to use a measuring tape to assess fundal height and knowing the time period } \\
\text { within which ultrasound dating is accurate } \\
\text { - Enable providers to build rapport and trust with clients and communities to encourage timely care-seeking and } \\
\text { open discussion of menstruation and sexual activity } \\
\text { - Providers will value gestational age estimates and be willing to invest time and energy into improving their accuracy } \\
\text { and documentation when they know that gestational age estimates are important to client health } \\
\text { - Provide respectful and high-quality maternal healthcare to increase the value of early and regular ANC for women }\end{array}$ \\
\hline Patient characteristics & $\begin{array}{l}\text { - Encourage women to seek first ANC as early as possible through outreach, awareness raising, and incentives since } \\
\text { this will improve LMP recall for those with regular menstruation prior to pregnancy and will enable early ultrasound } \\
\text { when gestational age estimation is most accurate } \\
\text { - Build health system capacity to communicate effectively across language groups and among migrants through } \\
\text { translation, appropriate within-community outreach efforts, and recruiting and training health care providers from } \\
\text { linguistically diverse communities } \\
\text { - Increase family support for early ANC and ultrasound by reducing financial and geographical barriers to access and } \\
\text { communicating the value of accurate gestational age estimates } \\
\text { - In addition to broadly increasing women's access to education, engage lower-literacy women through audio visual } \\
\text { media, community outreach and social groups to build understanding of menstruation and maternal healthcare } \\
\text { - Equip frontline health care providers with strategies to interpret dates reported by women with different cognitive } \\
\text { approaches to time, such as by linking harvests and festivals to the Gregorian calendar }\end{array}$ \\
\hline
\end{tabular}

Gestational age estimation • Interventions to improve gestational age accuracy must account for the inherent limitations in terms of their method accuracy and the stage in pregnancy when they can be used

- Ease of use and equipment required must be considered when considering strategies to improve gestational age estimation

community groups [35], as well as media campaigns, financial incentives, and home visits by healthcare providers [36].

This study provides a strong qualitative exploration of how healthcare providers working in rural government facilities or those with knowledge of the those facilities report their practices and experiences with gestational age estimation, documentation and use. Future research that explores client perspectives would provide valuable triangulation. Moreover, additional mixed methods research could better understand associations between specific provider characteristics (such as years of experience, cadre type, whether they are from the same region as their clients or not, gender, and work environment including facility type, client load, and staffing levels) and their attitudes and approaches to gestational age estimation. Finally, although ultrasound is closely regulated in India through the 2003 Pre-Conception and Pre-Natal Diagnostic Techniques (Prohibition Of Sex Selection)
Act [37] this study did not explore the potential link between regulation and availability. Future research is needed to understand whether efforts to curtail fetal sex determination have contributed to poor ultrasound access.

\section{Conclusions}

Frontline health care providers in Rajasthan's rural government facilities estimate gestational age using the methods most available to them and their clients. However, they receive little to no feedback on the accuracy of these assessments. Moreover, the gestational age estimates that they generate and document are not uniformly used to inform time-sensitive interventions. The limitations inherent to the estimation methods, and women's difficulty in recalling their last menstrual period, limit gestational age estimation to the nearest month rather than week. Despite the widespread use of time-sensitive interventions including antenatal corticosteroids, providers consider the 
existing processes to be sufficient. Interventions to improve the accuracy of gestational age estimation should consider factors at four levels: health system, frontline provider, clients, and the gestational age methods themselves.

\section{Supplementary information}

Supplementary information accompanies this paper at https://doi.org/10. 1186/s12884-020-03201-6.

Additional file 1. Annexure 1

\section{Abbreviations}

ANC: Antenatal care; ANM: Auxiliary nurse midwife; ASHA: Accredited social health activist; EDD: Estimated date of delivery; IP: Intrapartum care; Kl: Key informant; LMICs: Low- and middle-income countries; LMP: Last menstrual period; MBBS: Bachelor of Medicine, Bachelor of Surgery; MO: Medical officer; MoHFW: Ministry of Health and Family Welfare

\section{Acknowledgements}

We thank the healthcare providers and key informants for generously giving their time and insight to this study. We also acknowledge Brianne Kallam for her support, and Sudhakar Gaur, Rohit Bajpai, Meena Pahwa, Rupam, Rinki Gour, and Sanju Dixit, for their work on qualitative research management, data collection and translation.

\section{Authors' contributions}

KS, LN, EW, and SG developed the study protocol and data collection tools. UVS supported study design and sampling decisions. KS analyzed and interpreted the data and wrote the manuscript. MA provided critical feedback on the manuscript. All authors read and approved the final manuscript.

\section{Funding}

This study is made possible by the generous support of the American people through the United States Agency for International Development (USAID) under the terms of the Cooperative Agreement AID-OAA-A-1400028. The contents are the responsibility of the authors and do not necessarily reflect the views of USAID or the United States Government. USAID had no role in the design of the study and collection, analysis, and interpretation of data and in writing the manuscript.

\section{Availability of data and materials}

The datasets generated and analysed during the current study are not publicly available due the risk of identifying details of the respondents being gleaned through close reading of complete transcripts of the interviews. Relevant sections of the data, such as complete code reports and large portions of transcripts, are available from the corresponding author on reasonable request.

\section{Ethics approval and consent to participate}

Ethical approval was received from the Johns Hopkins Bloomberg School of Public Health Institutional Review Board and Sigma, India. All respondents provided written informed consent before participation.

\section{Consent for publication}

Not applicable.

\section{Competing interests}

The authors declare that they have no competing interests.

\section{Author details}

'USAID's Maternal and Child Survival Program/Johns Hopkins Bloomberg School of Public Health, Baltimore, USA. ${ }^{2}$ USAID's Maternal and Child Survival Program/Jhpiego, Baltimore, USA. ${ }^{3}$ USAID's Maternal and Child Survival Program/USAID, Washington, D.C., USA. ${ }^{4}$ Sigma, Delhi, India.
Received: 22 March 2020 Accepted: 21 August 2020

Published online: 11 September 2020

\section{References}

1. Blencowe $H$, Cousens $S$, Oestergaard MZ, Chou D, Moller AB, Narwal R, et al. National, regional, and worldwide estimates of preterm birth rates in the year 2010 with time trends since 1990 for selected countries: a systematic analysis and implications. Lancet. 2012;379(9832):2162-72.

2. Liu L, Oza S, Hogan D, Chu Y, Perin J, Zhu J, et al. Global, regional, and national causes of under-5 mortality in 2000-15: an updated systematic analysis with implications for the sustainable development goals. Lancet. 2016;388(10063):3027-35.

3. March of Dimes, PMNCH, Save the Children, WHO. In: Howson C, Kinney M, Lawn J, editors. Born Too Soon: The Global Action Report on Preterm Birth. Geneva: World Health Organisation; 2012.

4. WHO. WHO recommendation on antenatal corticosteroid therapy for women at risk of preterm birth from 24 weeks to 34 weeks of gestation (November 2015). Geneva; 2015.

5. Lefevre A, Agarwal S, Chamberlain S, Scott K, Godfrey A, Chandra R, et al. Are stage-based health information messages effective and good value for money in improving maternal newborn and child health outcomes in India? Protocol for an individually randomized controlled trial. Trials. 2019; 20(1):1-12.

6. Peter J, Benjamin P, LeFevre A, Barron P, Pillay Y. Taking digital health innovation to scale in South Africa: ten lessons from MomConnect. BMJ Glob Heal. 2018;3(Suppl 2):e000592.

7. Butt K, Lim K. Determination of gestational age by ultrasound, SOGC Clinical Practice Guidelines. J Obstet Gynaecol Canada. 2014;303:171-81.

8. Stebbins B, Jaffe R. Fetal biometry and gestational age estimation. In: Jaffe R, Bui T-H, editors. Textbook of Fetal Ultrasound. New York: Pantheon Publishing Group; 1999. p. 47-57.

9. ACOG Committee on Practice Bulletins. American College of Obstetrics and Gynecology Practice Bulletin No. 58. Ultrasonography in pregnancy. Obstet Gynecol. 2004;104:1449-58.

10. Lynch CD, Zhang J. The research implications of the selection of a gestational age estimation method. Paediatr Perinat Epidemiol. 2007 Sep; 21(s2):86-96.

11. Lee AC, Panchal P, Folger $L$, Whelan $H$, Whelan $R$, Rosner B, et al. Diagnostic accuracy of neonatal assessment for gestational age determination: a systematic review. Pediatrics. 2017;140(6):e20171423.

12. Papageorghiou AT, Ohuma EO, Gravett MG, Hirst J, da Silveira MF, Lambert A, et al. International standards for symphysis-fundal height based on serial measurements from the Fetal Growth Longitudinal Study of the INTE RGROWTH-21 st Project: prospective cohort study in eight countries. BMJ. 2016;7:15662.

13. Briand V, Saal J, Ghafari C, Huynh B-T, Fievet N, Schmiegelow C, et al. Fetal growth restriction is associated with malaria in pregnancy: a prospective longitudinal study in Benin. J Infect Dis. 2016 Apr 18;214(3):417-25.

14. White LJ, Lee SJ, Stepniewska K, Simpson JA, Dwell SLM, Arunjerdja R, et al. Estimation of gestational age from fundal height: a solution for resourcepoor settings. J R Soc Interface. 2012;9(68):503-10.

15. MoHFW India. National Family Health Survey 4 (2015-2016): state fact sheet Rajasthan [internet]. Mumbai; 2017.

16. Kozarsky PE, Harvey P. Chapter 10: India. In: CDC's Yellow Book. Center for Disease Control; 2020

17. Guest G, Johnson L. How many interviews are enough? An experiment with data saturation and variability. Field Methods. 2006;18(1):59-82.

18. Attride-Stirling J. Thematic networks: an analytic tool for qualitative research Qual Res. 2001;1(3):385-405.

19. ACOG. Methods for estimating the due date. Committee Opinion No. 700 Obs Gynecol. 2017;129:e150-4.

20. MoHFW India. Guidelines for primary health Centres [internet]. New Delhi; 2012.

21. MoHFW India. Guidelines for district hospitals [internet]. 2012

22. Rahman MM, Pallikadavath S. How much do conditional cash transfers increase the utilization of maternal and child health care services? New evidence from Janani Suraksha Yojana in India. Econ Hum Biol. 2018:31:164-83.

23. Kim ET, Singh K, Moran A, Armbruster D, Kozuki N. Obstetric ultrasound use in low and middle income countries: a narrative review. Reprod Health. 2018;15(1):1-26. 
24. Gonzaga MA, Kiguli-Malwadde E, Businge F, Byanyima RK. Utilisation of obstetric sonography at a peri-urban health Centre in Uganda. Pan Afr Med J. 2010;7(24):24.

25. Srivastava A, Avan BI, Rajbangshi P, Bhattacharyya S. Determinants of women's satisfaction with maternal health care: a review of literature from developing countries. BMC Pregnancy Childbirth. 2015 Dec 18;15(1):97.

26. Anand T, Garg S. Menstruation related myths in India: strategies for combating it. J Fam Med Prim Care. 2015;4(2):184.

27. Hennegan J, Shannon AK, Rubli J, Schwab KJ, Melendez-Torres GJ. Women's and girls' experiences of menstruation in low- and middle-income countries: A systematic review and qualitative metasynthesis. Myers JE. PLOS Med. 2019;16(5):e1002803.

28. Grum T, Brhane E. Magnitude and factors associated with late antenatal care booking on first visit among pregnant women in public health centers in central zone of Tigray Region, Ethiopia: A cross sectional study. Figueras A. PLoS One. 2018;13(12):e0207922.

29. Ebonwu J, Mumbauer A, Uys M, Wainberg ML, Medina-Marino A. Determinants of late antenatal care presentation in rural and peri-urban communities in South Africa: A cross-sectional study. El Bcheraoui C. PLoS One. 2018;13(3):e0191903.

30. Ewunetie AA, Munea AM, Meselu BT, Simeneh MM, Meteku BT. DELAY on first antenatal care visit and its associated factors among pregnant women in public health facilities of Debre Markos town, North West Ethiopia. BMC Pregnancy Childbirth. 2018;18(1):173.

31. Jacobs C, Michelo C, Moshabela M. Why do rural women in the most remote and poorest areas of Zambia predominantly attend only one antenatal care visit with a skilled provider? A qualitative inquiry. BMC Health Serv Res. 2018;18(1):1-9.

32. Simkhada B, Van Teijlingen ER, Porter M, Simkhada P. Factors affecting the utilization of antenatal care in developing countries: systematic review of the literature. J Adv Nurs. 2008;61(3):244-60.

33. Myer L, Harrison A. Why do women seek antenatal care late? Perspectives from rural South Africa. J Midwifery Womens Health. 2003 Jul 8;48(4):26872.

34. Griffiths P, Stephenson R. Understanding users' perspectives of barriers to maternal health care use in Maharashtra. India J Biosoc Sci. 2001;33(3):33959.

35. Manandhar DS, Osrin D, Shrestha BP, Mesko N, Morrison J, Tumbahangphe $\mathrm{KM}$, et al. Effect of a participatory intervention with women's groups on birth outcomes in Nepal: cluster randomised controlled trial. Lancet. 2004; 364(9438):970-9 [2015 Oct 29].

36. Mbuagbaw L, Medley N, Darzi AJ, Richardson M, Habiba Garga K, OngoloZogo P. Health system and community level interventions for improving antenatal care coverage and health outcomes. Cochrane Database Syst Rev. 2015 Dec 1;1(12):1-157.

37. Mani S. Guidelines for ultrasound owners and owners of clinics, diagnostic centres, nursing homes and hospitals. Indian J Radiol Imaging. 2012;22(2): 125.

\section{Publisher's Note}

Springer Nature remains neutral with regard to jurisdictional claims in published maps and institutional affiliations.

Ready to submit your research? Choose BMC and benefit from:

- fast, convenient online submission

- thorough peer review by experienced researchers in your field

- rapid publication on acceptance

- support for research data, including large and complex data types

- gold Open Access which fosters wider collaboration and increased citations

- maximum visibility for your research: over $100 \mathrm{M}$ website views per year

At $\mathrm{BMC}$, research is always in progress.

Learn more biomedcentral.com/submissions 